
allemande

51-2| 2019

Les Humanités environnementales : circulations et renouvellement des savoirs en France et en Allemagne

Michel FABRÉGUET et Danièle HENKY (dir.), Mémoires et représentations de la déportation dans l'Europe contemporaine

Paris, L'Harmattan, 2015

\title{
Ségolène Plyer
}

\section{OpenEdition \\ Journals}

Édition électronique

URL : https://journals.openedition.org/allemagne/2105

DOI : 10.4000/allemagne.2105

ISSN : 2605-7913

Éditeur

Société d'études allemandes

Édition imprimée

Date de publication : 10 décembre 2019

Pagination : 533-535

ISSN : 0035-0974

Référence électronique

Ségolène Plyer, « Michel fabréguet et Danièle henky (dir.), Mémoires et représentations de la déportation dans l'Europe contemporaine », Revue d'Allemagne et des pays de langue allemande [En ligne], 51-2 | 2019, mis en ligne le 02 décembre 2019, consulté le 23 mai 2021. URL : http://journals.openedition.org/ allemagne/2105; DOI : https://doi.org/10.4000/allemagne.2105 
Michel FABRÉGUET et Danièle HENKY (dir.), Mémoires et représentations de la déportation dans l'Europe contemporaine, Paris, L'Harmattan (coll. Inter-National), $2015,234 p$.

À l'heure où disparaissent les derniers témoins des déportations de la Seconde Guerre mondiale, quelles traces cette expérience laisse-t-elle en Europe? En se saisissant de cette question, l'ouvrage collectif et interdisciplinaire dirigé par M. Fabréguet et D. Henky (Université de Strasbourg) fait le pari d'enrichir l'histoire européenne du phénomène de la déportation, vu après 1945 au travers d'un prisme national insistant sur les prisonniers de guerre et les résistants, puis éclairé par la prise de conscience de l'importance et de la spécificité des déportations raciales menées par le Troisième Reich.

Dans la première partie, Michel Fabréguet montre, en comparant des témoignages de l'immédiat après-guerre avec les discours de grands résistants prononcés à la fin de la Guerre froide, que les déportés avaient une vision d'abord nationale des relations internationales, marquée par les expériences des années 1930, ou bien une vision universaliste "naïve»; retrouver, dans l'épreuve de la déportation, une solidarité entre déportés au nom de valeurs européennes tient de la reconstruction a posteriori. Marcel Tambarin s'interroge sur le passage à la mémoire non communicationnelle en Allemagne fédérale, tandis que Nadine Willmann déchiffre, dans les mémoires d'anciens détenus politiques après 1933, le processus assimilant progressivement les prisons allemandes au régime extra-judiciaire des camps. Nathanaël Wadbled et Michèle Farache s'attaquent à la représentation de l'irreprésentable: visiter Auschwitz est comme affronter la méduse. Le musée a pour rôle de conduire et de protéger le visiteur, comme le bouclier de Persée. Riche et intéressant, cet article ne remet néanmoins pas en question le présupposé selon lequel le visiteur saurait d'avance ce qui va lui être montré et aurait déjà pris la mesure inouie de l'expérience annihilatrice dont il vient voir les traces à Oświęcim. Puis Gilbert Merlio retrace les débats des années 1990 en Allemagne sur la muséalisation des prisons des services secrets soviétiques, souvent installées dans les anciens camps nazis après 1945.

Cette étude des mots et des musées est suivie d'une deuxième partie consacrée à l'expression artistique de la déportation, centrée sur le témoignage qui se décline en trace et document, avertissement, remémoration, mise en présence... Les vitraux étudiés par Jean-Claude Lescure, ayant pour thème les prisonniers de guerre exilés, voire la déportation de résistants, se rapprochent de l'ex-voto. Leur fréquence et leur diffusion sur tout le territoire français permettent de mesurer l'ample écho trouvé par la déportation de membres de la communauté catholique. Mais c'est bien la Shoah qui stimule le plus la réflexion sur la fonction du témoignage et sa possibilité même. À travers la succession des médias qui veulent en rendre compte depuis 1945 (par la photographie, puis la mise en mots, enfin par l'cuvre d'art qui peut être partiellement documentaire), Jérôme Moreno explique les fonctions sociales successives du témoignage sur l'activité concentrationnaire et génocidaire nazie, en mettant en évidence les relations entre le medium choisi et la progressive prise de conscience des crimes du Troisième Reich. Alessandro Carrieri revient aux œuvres musicales composées en situation de déportation, notamment à L'Empereur d'Atlantis de Viktor Ullmann, ceuvre très connue et commentée, dont il rappelle sommairement le caractère désespérément protestataire, encore sensible grâce à sa construction dramatique et à ses citations d'autres grands compositeurs. Régine Atzenhoffer retrouve le propos de Claude Lanzmann dans Shoah (1985) qui, en excluant presque complètement le recours à la fiction et aux images d'archives, redonne aux lieux, visages, silences et mots de témoins la 
possibilité de "dire vrai» sur le génocide au-delà du mensonge institutionnalisé par les nazis $^{(3)}$ : Shoah "n'est pas un film de souvenirs, mais un film de la mémoire», transformant les "spectateurs que nous sommes [en] des témoins de l'Holocauste», c'est-à-dire des "témoins d'un 'événement-sans-témoin'» (p. 138). Martine Benoît livre un résumé sensible de sa source, en l'occurrence un roman de Peter Edel qui, au début des années 1970, aborde la question d'une mémoire juive spécifique et de ses difficultés à se faire reconnaître au sein de l'antifascisme est-allemand.

La troisième partie s'interroge sur l'usage du témoignage, parallèlement à la transmission du savoir scolaire sur les déportations de la Seconde Guerre mondiale. Cathy Leblanc explique qu'un tel savoir ne pourra être intériorisé comme une expérience digne de mémoire, donc liée à l'univers affectif des jeunes, que si, d'une part, l'empathie des élèves est sollicitée et que, d'autre part, ils acceptent de ne pas tout comprendre d'un phénomène dont l'inhumanité résiste à l'explication. Danièle Henky analyse le paradoxe de la littérature de jeunesse qui, dès la fin des années 1940, s'est donné pour objectif de "transmettre 'une expérience devenue héritage de l'inhumain'» ${ }^{(4)}$ à travers des formes souvent stéréotypées, mais utilisées avec sensibilité par des auteurs "passeurs de mémoire soucieux d'aider l'enfant à devenir citoyen» ${ }^{(5)}$, en l'aidant à se recentrer sur des émotions accessibles, plutôt qu'en se perdant dans la monstruosité du mythe. Le théâtre de marionnettes étudié par Maja Saraczynska-Laroche a le même souci de recourir au transfert ou au détournement «de cette question jugée irreprésentable, notamment à un public jeune » ${ }^{(6)}$, mais à laquelle les auteurs étudiés ne renoncent pas pour des raisons autobiographiques. Elle met en lumière l'inventivité poignante de ces derniers qui, incapables d'oublier ce traumatisme, utilisent par exemple une marionnette comme le prolongement de leur corps pour jouer les scènes où le déporté est déshumanisé. Enfin, Stanislas Hommet et Jan Lofström présentent une partie des résultats du projet de recherche commun aux universités de Caen et d'Helsinki, Teaching history for Europe in common (projet THIEC, 2008-2012), qui a interrogé des lycéens de plusieurs pays européens sur des épisodes du "passé douloureux» de leur pays, liés aux déportations de la Seconde Guerre mondiale. Les réactions des enquêtés montrent davantage de références à une culture civique générale qu'à une historicisation de cette culture qui identifierait clairement les bourreaux et rendrait acceptable l'idée d'une réparation matérielle des violences subies. Leur mémoire, sans être abstraite (ils compatissent avec de vraies victimes), élude la question de la responsabilité individuelle des injustices historiques. Néanmoins, la plupart accepte l'idée d'une continuité entre ce passé douloureux et le présent et ne rejette pas cet héritage.

À la lecture de ce riche ensemble, on regrette de devoir naviguer sans boussole entre les termes polysémiques de mémoire, déportation, témoignage, dont les définitions successives ne sont pas expliquées, mises en rapport ou contextualisées à l'échelle du volume. Le rapport entre la Shoah et les autres expériences de déportation souffre de n'être pas explicité, alors même que l'ouvrage fait ressortir la différence des mémoires, voire l'incompatibilité de leurs enjeux. Contrairement à ce que laisse supposer le titre,

3 Régine ATZENHOFFER, "De la difficulté de représenter dans Shoah de C. Lanzmann ou $9 \mathrm{~h} 30$ de mise en images de la parole de témoins», p. 127-138, ici p. 128.

4 Lucie CAmpos, Fictions de l'après: Coetzee, Kertész, Sebald. Temps et contre-temps de la conscience historique, Paris, Classiques Garnier, 2012, p. 202, cité par Danièle HENKY dans "L'indicible histoire de la Shoah: du travail de mémoire et d'écriture en littérature de jeunesse», p. 169-182, ici p. 169.

5 D. HENKY, ibid., p. 182.

6 Maja SARACZYNSKA-LAROCHE, «Représentation théâtrale de la déportation: détournement par la pratique du théâtre de marionnettes», p. 183-190, ici p. 183. 
les contributions ne s'intéressent que rarement aux mémoires collectives des différents groupes sociaux et nationaux en Europe, mais portent bien plus souvent sur le témoignage. À travers l'étude de témoignages souvent individuels ou produits par un petit groupe, hélas rarement analysés dans leur contexte de production et de réception, elles rejoignent les problématiques bien connues des rapports entre mémoire et oubli, du deuil et de l'incapacité d'oublier, de l'injonction de la remémoration et du problème de la transmission. On aurait aimé que l'originalité de ce recueil d'analyses, moins historiques que littéraires et artistiques, s'affirme davantage en remplissant plus systématiquement les objectifs fixés page 11, notamment celui de l'étude des moyens techniques et stylistiques de représentation. En outre, la présentation des corpus, des périodes et des lieux étudiés étant souvent rapide, il est difficile de s'interroger sur les transferts ou les circulations de ces mémoires. Si la dimension européenne est assurée par quelques articles capables d'analyser avec justesse un regard non français sur la déportation, seul le dernier article présente explicitement la problématique pourtant actuelle de l'élargissement des mémoires européennes à l'Europe de l'Est. Des expériences de déportation qui y ont été fréquentes, comme le travail forcé dans le Reich, ne sont curieusement pas abordées. D'autres (celle des Tsiganes par exemple) sont sous-représentées.

Ces réserves mises à part, la mosaïque d'expériences littéraires et artistiques qui se forme au fil du volume - comportant les reproductions des vitraux cités par JeanClaude Lescure, se terminant par un éloge de feu Pierre Ayçoberry et un résumé des contributions - ne manque pas d'intérêt, notamment lorsqu'il s'agit d'études de cas à partir de sources de première main. Certains auteurs parviennent à communiquer leur passion pour les formes que prend la transmission de l'expérience de la déportation; formes diverses, presque braconnières parfois, insérées pour certaines dans des pratiques purement locales (comme les vitraux d'églises) ou mineures (la littérature de jeunesse). De la lecture de cet ouvrage, on retire aussi plusieurs belles formules, forgées ou mises en exergue par les auteurs. Leurs textes témoignent de la réalité d'une expérience plurielle, dont on regrette néanmoins de ne pas bien saisir l'unité postulée par les directeurs du recueil et dont la généralisation de la mémoire à l'échelle européenne, bien qu'elle ne fasse pas de doute, mériterait encore d'être creusée.

Ségolène Plyer

Gwénola SEBAuX (dir.), Identités, migrations et mobilités transnationales. Europe (XIXe-XX|e siècle). Étude de cas: Allemagne, Autriche, Roumanie, France, Israël, Villeneuve d'Asca, Presses universitaires du Septentrion, 2017, 234 p.

Les mobilités, c'est bien connu, sont l'antithèse de la stabilité dont les identités collectives ont besoin pour se constituer et qui, pour les plus abouties, s'appuient sur l'État et ses moyens administratifs et coercitifs, ces derniers incluant le contrôle des déplacements de population. Néanmoins, l'histoire contemporaine de l'Europe continentale ou projetée outre-mer, étudiée ici au travers d'exemples allemands, autrichiens et roumains, français et israéliens, est en contradiction avec cette profession de stabilité. Cette contradiction est passée au crible par dix-sept études de cas qui, des Empires à la construction européenne en passant par l'établissement ou l'approfondissement des États-nations au XXe siècle, analysent les tensions entre identités collectives, États 